\title{
Towards a Viscous Wall Model for Immersed Boundary Methods
}

\author{
Christoph Brehm*1,2 \\ ${ }^{1}$ Science and Technology Corporation, Moffett Field, CA 94035 \\ ${ }^{2}$ AME Department, University of Arizona, Tucson, AZ \\ Michael F. Barad ${ }^{\dagger 3}$, and Cetin C. Kiris ${ }^{\ddagger 3}$ \\ ${ }^{3}$ NASA Ames Research Center, Moffett Field, CA 94035
}

\begin{abstract}
Immersed boundary methods are frequently employed for simulating flows at low Reynolds numbers or for applications where viscous boundary layer effects can be neglected. The primary shortcoming of Cartesian mesh immersed boundary methods is the inability of efficiently resolving thin turbulent boundary layers in high-Reynolds number flow application. The inefficiency of resolving the thin boundary is associated with the use of constant aspect ratio Cartesian grid cells. Conventional CFD approaches can efficiently resolve the large wall normal gradients by utilizing large aspect ratio cells near the wall. This paper presents different approaches for immersed boundary methods to account for the viscous boundary layer interaction with the flow-field away from the walls. Different wall modeling approaches proposed in previous research studies are addressed and compared to a new integral boundary layer based approach. In contrast to common wall-modeling approaches that usually only utilize local flow information, the integral boundary layer based approach keeps the streamwise history of the boundary layer. This allows the method to remain effective at much larger $y^{+}$values than local wall modeling approaches. After a theoretical discussion of the different approaches, the method is applied to increasingly more challenging flow fields including fully attached, separated, and shock-induced separated (laminar and turbulent) flows.
\end{abstract}

\section{Introduction}

Immersed Boundary Methods (IBMs) have been developed for many years and have appeared in various forms since they were first introduced by Peskin ${ }^{1,2}$ (see for example Goldstein et al. ${ }^{3}$ LeVeque and Li, ${ }^{4}$ Wiegmann and Bube, ${ }^{5}$ Linnick and Fasel, ${ }^{6}$ Johansen and Colella, ${ }^{7}$ Mittal and Iaccarino, ${ }^{8}$ Zhong, ${ }^{9}$ Duan et $a l .{ }^{10}$ and many others). These methods were first introduced as a nontraditional approach for numerically solving initial/boundary-value problems for complex geometries on Cartesian meshes and have matured to become increasingly important for a wide range of applications. ${ }^{1-13}$ Figure 1 shows a 2D example of an arbitrary immersed boundary cutting through a Cartesian grid. One of the key advantages of immersed boundary methods is that the computational meshes can be automatically generated starting from a water tight surface triangulation independent of the complexity of the geometry. The grid-generation process for body-fitted structured or unstructured grids for complex geometries is generally very laborious. The process aims at generating well behaved grids that have sufficient local resolution while minimizing the total number of required grid points. Except for simple geometries, these conflicting requirements can lead to deterioration in grid quality, thereby negatively impacting the accuracy and convergence properties of the flow solver. For highly complex geometries, which are relevant for many fields of science and engineering, the process of generating a high-quality grid is extremely time consuming. For flows involving moving and deforming boundaries, IBMs also provide clear advantages over classical body-fitted grid approaches. Simulating such

\footnotetext{
${ }^{*}$ Research Professor, Research Scientist, and AIAA Senior Member

${ }^{\dagger}$ Research Scientist, Computational Aerosciences Branch, NAS Division, MS N258-2 and AIAA Senior Member

${ }_{\ddagger}^{\ddagger}$ Branch Chief, Computational Aerosciences Branch, NAS Division, MS N258-2 and AIAA Senior Member
} 
flows on body-fitted grids generally requires generating a new grid at each time step and a procedure to project the solution onto this new grid (Tezduyar ${ }^{14}$ ). These two characteristics (grid deformation and projection of solution) for body-fitted grids associated with simulating flows with moving and deforming boundaries may negatively impact the accuracy, robustness, and computational cost of the numerical solution method. Particularly, in cases where the boundary exhibits large motions/deformations, body-conformal grid strategies cause immense difficulties in the solution procedure. This is where an immersed boundary approach with its fully Eulerian approach provides great advantages. IBMs provide a much more convenient way of including the body motion and deformation by using a stationary non-deforming Cartesian grid. While these methods simplify the grid generation process, a detailed mathematical understanding of the IBM is necessary to avoid a negative impact of the boundary treatment on the robustness, the convergence behavior and the accuracy of the numerical scheme.

Immersed boundary methods also posses well known shortcomings that have limited their applicability to a wider range of applications. Most IBMs are only lower-order accurate and suffer from robustness issues when extended to higher-order. The near wall accuracy is, however, critical when simulating laminar to turbulent transition scenarios for wall-bounded flows in order to capture relevant instability mechanisms. Higher-order immersed boundary methods have been presented in a number of research studies by Linnick and Fasel, ${ }^{6}$ Zhong, ${ }^{9}$ Duan et al. ${ }^{10}$ Brehm and Fasel, ${ }^{15}$ Brehm et al.,${ }^{16}$ and others.

Moreover, for high-Reynolds number viscous flow simulations, current immersed boundary approaches are inefficient in resolving the viscous boundary layers since the Cartesian mesh layout generally does not allow the use of (wall-normal) high aspect ratio cells in the vicinity of the wall. This issue has been addressed in several research investigations, but the cur-

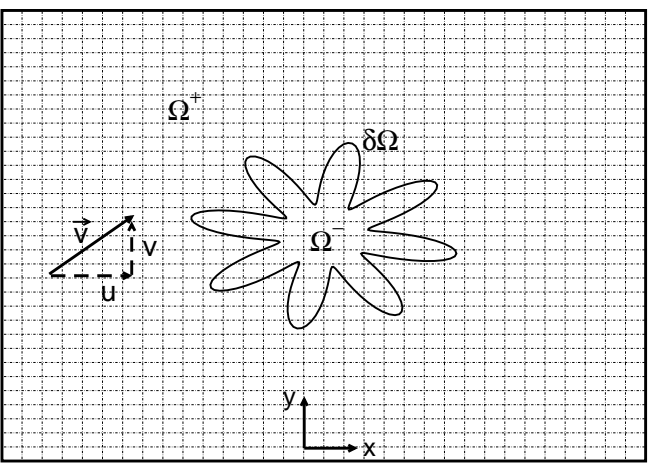

Figure 1. Arbitrary geometry immersed into a Cartesian grid. rent approaches, which are mainly based on the application of wall-functions near the immersed boundary, lack maturity. Ruffin and Lee ${ }^{17}$ used the standard $k$ - $\epsilon$ turbulence model by Launder and Spalding with Spalding's wall model formulation and combined it with an immersed boundary ghost-cell approach. They obtained a fair agreement for low Mach number, two-dimensional and axisymmetric test cases for $y^{+}$-values of up to 200. Berger and Aftosmis ${ }^{18}$ developed a method for simulating steady viscous compressible flows in 2D utilizing a Cartesian cut-cell finite volume approach. For low cell Reynolds numbers a quadratic wall model was employed and for high cell Reynolds numbers an analytic wall model was used that is based on the Spalart-Allmaras turbulence model. Good agreement with both theoretical and experimental data was obtained for flows over a flat plate and sub- and transonic airfoils. Berger and Aftosmis showed convergence of surface pressures with wall spacing of $0.1 \%$ chord and mesh converged skin friction for 2-4 times smaller grid spacings.

Another strategy to account for viscous effects in an immersed boundary method is to couple the immersed boundary Euler or Navier-Stokes solver with an integral boundary layer (IBL) method. More than two decades ago, Drela ${ }^{19}$ demonstrated the great potential of this approach for $2 \mathrm{D}$ sub- and supersonic airfoil flows. Drela solved the Euler equations on a conservative streamline grid (not immersed) and coupled this solver to a two-equation integral boundary-layer formulation. A strong coupling was obtained using the displacement thickness concept. Aftosmis et al. ${ }^{20}$ and Rodriguez et al. ${ }^{21}$ followed up on this approach and utilized transpiration boundary conditions to account for the boundary-layer displacement thickness in a cut-cell, multi-level Cartesian-mesh Euler solver. For 3D flows, the boundary conditions were obtained on $2 \mathrm{D}$ strips where the 2D boundary-layer equations can be solved. Between the 2D strips the solution was re-distributed by simple interpolation or solving a Poisson equation on the surface triangulation. Aftosmis et al. obtained good agreement with flight data for the RM-10 vehicle by applying decoupled viscous corrections to the inviscid solution. They also demonstrated coupled-IBL simulation capabilities for the unit span RAE 2822 airfoil, Onera M6, and the DLR F-4 wing-body yielding good agreement with experimental data.

For lattice Boltzmann methods (LBMs), it is common practice to apply wall boundary conditions in an immersed boundary fashion. The success of this method in the past decade (see for example Powerflow by EXA Corporation) appears to be associated with an increased computational efficiency (at least for secondorder solutions) in terms of the operation-count per lattice point in comparison to conventional (FD, FV, 
and FEM) approaches. Nevertheless, for high-Reynolds number flows, LBMs commonly still require a wall modeling-type approach because even for the increased efficiency of these methods viscous walls can rarely be resolved to levels of $y+<10$. To the extend of what is being published about these methods, the wall model-type approaches for LBMs are also commonly based on local flow information and, therefore, require grid resolutions of $y^{+}=10-100$. Considering the remaining challenges for the different approaches it is fair to conclude that an overall satisfying solution for high-Reynolds number flows has not been found yet.

The current paper aims at advancing the state-of-the-art in viscous wall modeling for immersed boundary methods. The method is implemented in LAVA's Cartesian grid solver employing the immersed boundary method by Brehm et al. ${ }^{15,16}$ While some of the intricacies with respect to this particular immersed boundary method are being addressed, the viscous wall model can basically be employed without major modifications to any of the aforementioned immersed boundary methods or LBMs. The fundamental idea of viscous wall modeling-type approaches is to account for the viscous boundary layer interacting with the solution away from the walls. In order to allow for acceptable flow solutions at large $y^{+}$values, the authors found that it is crucial to keep the boundary layer history in the streamwise direction and not only rely on local flow quantities. The basic idea is to combine an integral boundary layer solver with an immersed boundary method. Since some of the grid points are located in the boundary layer, and unsteady flows are targeted with this solver, a fully interactive IBL-IBM approach is taken.

The current abstract provides an overview of what can be expected in the final paper. The abstract proceeds as follows: section II introduces the numerical scheme and the immersed boundary method used to solve the compressible Navier-Stokes equations. Section III presents three types of viscous wall models with increasing fidelity used to account for boundary layer effects in immersed boundary methods. Section IV provides an overview and some preliminary results of well-documented test cases to compare the accuracy of the different methods. Finally, some preliminary conclusions and an outlook to the final paper is provided in section $\mathrm{V}$.

\section{Numerical Methods}

\section{II.A. Interior and Temporal Discretizations}

The compressible Navier-Stokes equations considering an ideal, Newtonian, non-reactive gas are

$$
\begin{gathered}
\frac{\partial \rho}{\partial t}+\nabla \cdot(\rho \boldsymbol{u})=0, \\
\frac{\partial \rho \boldsymbol{u}}{\partial t}+\nabla \cdot(\rho \boldsymbol{u} \boldsymbol{u}+p \underline{\boldsymbol{\delta}}-\underline{\boldsymbol{\tau}})=0, \\
\frac{\partial E}{\partial t}+\nabla \cdot(E \boldsymbol{u}+\boldsymbol{u} \cdot(p \underline{\boldsymbol{\delta}}-\underline{\boldsymbol{\tau}})-\kappa \nabla T)=0, \quad \text { with } \\
E=\frac{p}{\gamma-1}+\frac{1}{2} \rho \boldsymbol{u} \cdot \boldsymbol{u} \quad \text { and } \quad p=\rho R T .
\end{gathered}
$$

In the above equations $\rho$ is the density, $\boldsymbol{u}$ is the velocity vector, $\underline{\boldsymbol{\delta}}$ is the unit tensor, $p$ is the static pressure, $T$ is the temperature, $E$ is the total energy, $\gamma$ is the specific heat ratio, $R$ is the gas constant, and $\kappa$ is the thermal conductivity. The viscous stress tensor is

$$
\underline{\boldsymbol{\tau}}=\mu(2 \underline{\boldsymbol{S}})+\left(\beta-\frac{2}{3} \mu\right)(\nabla \cdot \boldsymbol{u}) \underline{\boldsymbol{\delta}} .
$$

The dynamic viscosity is given by $\mu, \beta$ is the bulk viscosity, and the strain rate tensor $\underline{\boldsymbol{S}}$ is defined as $\underline{\boldsymbol{S}}=\frac{1}{2}\left(\nabla \boldsymbol{u}+(\nabla \boldsymbol{u})^{T}\right)$.

Equations (1)-(2) can be re-written in vector form as follows

$$
\frac{\partial \boldsymbol{W}}{\partial t}+\nabla \cdot\left(\overrightarrow{\mathcal{F}}-\overrightarrow{\mathcal{F}}_{v}\right)=0, \quad \text { with } \quad \overrightarrow{\mathcal{F}}=\left(\boldsymbol{F}^{1}, \boldsymbol{F}^{2}, \boldsymbol{F}^{3}\right) \quad \text { and } \quad \overrightarrow{\mathcal{F}}_{v}=\left(\boldsymbol{F}_{v}^{1}, \boldsymbol{F}_{v}^{2}, \boldsymbol{F}_{v}^{3}\right) .
$$


The conservative variable vector $\boldsymbol{W}=(\rho, \rho u, \rho v, \rho w, \rho H-p)^{T}$ and the inviscid fluxes are

$$
\boldsymbol{F}^{1}=\left[\begin{array}{c}
\rho u \\
\rho u^{2}+p \\
\rho u v \\
\rho u w \\
\rho u H
\end{array}\right], \boldsymbol{F}^{2}=\left[\begin{array}{c}
\rho v \\
\rho v u \\
\rho v^{2}+p \\
\rho v w \\
\rho v H
\end{array}\right] \text {, and } \boldsymbol{F}^{3}=\left[\begin{array}{c}
\rho w \\
\rho w u \\
\rho w v \\
\rho w^{2}+p \\
\rho w H
\end{array}\right],
$$

where the total enthalpy is $H=h+\vec{u} \cdot \vec{u} / 2$. The viscous fluxes are

$$
\boldsymbol{F}_{v}^{1}=\left[\begin{array}{c}
0 \\
\tau_{x x} \\
\tau_{x y} \\
\tau_{x z} \\
f_{v}^{1}
\end{array}\right], \boldsymbol{F}_{v}^{2}=\left[\begin{array}{c}
0 \\
\tau_{y x} \\
\tau_{y y} \\
\tau_{y z} \\
f_{v}^{2}
\end{array}\right] \text {, and } \boldsymbol{F}_{v}^{3}=\left[\begin{array}{c}
0 \\
\tau_{z x} \\
\tau_{z y} \\
\tau_{z z} \\
f_{v}^{3}
\end{array}\right]
$$

The interior discretization of the convective terms follows the approach presented in Laible and Fasel. ${ }^{22}$ The forward-flux, $\mathbf{F}^{+}$, and the backward-flux, $\mathbf{F}^{-}$, are discretized with $n^{\text {th }}$-order accurate centered upwind finite differences as proposed by Zhong. ${ }^{23}$ As in Laible and Fasel, ${ }^{22}$ a parameter $\alpha$ is used to determine the degree of upwinding for the grid-centered upwind differences

$$
\left.\frac{\partial \phi}{\partial x}\right|_{i}=\sum_{k=i-N_{s}}^{i+N_{s}} c_{k} \phi_{k}-\left.\alpha \overline{\Delta x} \frac{\partial^{2 N_{s}-1} \phi}{\partial x^{2 N_{s}-1}}\right|_{i},
$$

where $c_{k}$ is the finite difference stencil coefficient, $\overline{\Delta x}$ is the averaged grid spacing over the spatial extent of the stencil and $N_{s}$ determines the number of grid points in the stencil. Note that a central difference stencil can be achieved by choosing $\alpha=0$. In this case, a higher-order filter based on work by Visbal and Gaitonde ${ }^{24}$ is used to regularize the solution. In accordance with the chosen discretization in Laible and Fasel, ${ }^{22}$ the $n^{\text {th }}$-order accurate upwind stencils are calculated with $n=9,7,5,3,1$ and $\alpha=-1500,72,-12,0.6,-1$. In the current paper, it is assumed that the numerical fluxes are approximated with flux vector splitting schemes, i.e., Lax Friedrichs, van Leer, AUSMPW+, etc. The compressible Navier-Stokes equations are advanced in time with an explicit fourth-order accurate Runge-Kutta scheme based on the Shu-Osher formulation. ${ }^{25}$ For accurately capturing flow discontinuities higher-order shock capturing schemes ${ }^{26}$ are also available. The computational domain is decomposed into block-structured sub-domains supporting adaptive-mesh refinement as well as generalized curvilinear coordinates. In order to maintain higher-order accuracy across coarse-fine block interfaces, up-to fourth-order accurate prolongation and restriction operators are available.

\section{II.B. Immersed Boundary Method}

In this section, the immersed boundary method presented in Brehm and Fasel ${ }^{15}$ is extended to the compressible Navier-Stokes equations. Many immersed methods have been developed in the past; however, in the derivation of these schemes, usually only the order of the local truncation error or accuracy of the numerical scheme has been considered. A posteriori, the numerical stability of these schemes is commonly demonstrated (in a global sense) by considering a number of different test-problems, and in a few cases, an additional global matrix stability analysis is employed (see for example Zhong ${ }^{9}$ ). The basic idea of the immersed boundary method by Brehm and Fasel ${ }^{15}$ is that the stencil coefficients are locally optimized in order to improve the stability of the scheme.

The notion of developing a strategy for improving the spectral properties of immersed methods originated from the idea that the stability of the numerical scheme can be formulated as an $N$-dimensional optimization problem, where $N$ represents the number of irregular grid points. Instead of solely considering the local truncation error in the derivation of the finite-difference grid stencil at an irregular grid point, an overdetermined system of equations is constructed to determine the stencil coefficients. An over-determined system of equations is employed because it allows to select free parameters, which can be tuned towards optimizing the local stability properties of the finite-difference stencil. Thus, assuming one free parameter per irregular grid point an $N$-dimensional optimization problem for $N$ irregular grid points is obtained. 
The objective function of the optimization problem must be carefully chosen and depends on the nature of the particular partial-differential equation of interest. For advection-diffusion-type equations, the spectral radius of the update matrix may be considered an appropriate objective function. In the current paper, we refer to the update matrix as the matrix which updates the solution from time $t_{n}$ to the next time-step, $t_{n+1}$. The update matrix, therefore, contains information about the spatial and temporal discretization of the numerical scheme. The spectral radius is well-suited in this situation, because its actual value is associated with the stability of the numerical scheme and it can be used to formulate a necessary condition for the stability of the update matrix. For non-linear systems of equations, a linearization is necessary prior to applying the outlined linear stability theory concepts.

In principle, it is possible to solve a coupled $N$-dimensional optimization problem for $N$ irregular grid points. From a practical point of view, however, it is not very efficient or desirable to solve such a large system of equations, especially for FSI problems where the grid topology is time dependent, thereby requiring this procedure to be repeated at each time-step. Hence, in the current approach, the boundary stencils are isolated from the remainder of the computational domain so that the optimization problem can be formulated for each irregular grid-point separately. Instead of solving a global $N$-dimensional optimization problem, $N$ 1D optimization problems are solved at each irregular grid point individually. This approach greatly reduces the computational effort needed to determine the "optimal" stencil coefficients. The aspect of turning the global $N$-dimensional problem into $N$ one-dimensional problems is referred to as "localization" or "localization assumption". In Brehm and Fasel, ${ }^{15}$ it was demonstrated, both numerically and analytically, that for advection-diffusion type equations, the localization assumption appears to be valid as long as the von Neumann number does not reach a limiting value. For a more detailed discussion on the original approach, the interested reader is referred to Brehm and Fasel. ${ }^{15}$ In the following discussion, the basic steps for extending this method to the compressible Navier-Stokes equations are outlined.

The main difference for the application of the IBM to the compressible Navier-Stokes equations instead of the incompressible Navier-Stokes equations (solved by utilizing the approximate projection method) is the use of numerical fluxes and elimination of the projection step. Flux vector splitting schemes, i.e. van Leer, ${ }^{27}$ Lax Friedrichs, and AUSMPW+, are adopted here. In the flux vector splitting approach, the convective flux is divided into a forward-flux and a backward-flux, $\mathbf{F}=\mathbf{F}^{-}+\mathbf{F}^{+}$. Flux vector splitting is also applied at irregular grid points, which can be employed to stabilize the irregular grid stencils. Assuming subsonic flow speeds in the vicinity of the immersed boundary (no slip wall), upwinding can always be applied in the upwind direction towards the wall. This is in contrast to the scalar advection equation where the advection speed determines the upwind direction. The basic ideas of the immersed boundary method are discussed for the 1D Euler equations in conservative variable form,

$$
\left(\begin{array}{c}
\rho \\
\rho u \\
E
\end{array}\right)_{t}=\left(\begin{array}{c}
\rho u \\
\rho u^{2}+p \\
u(E+p)
\end{array}\right)_{x},
$$

where the total energy can be expressed as $E=\rho\left(T /\left((\gamma-1) \gamma M^{2}\right)+u^{2} / 2\right)$. The fluid is assumed to be an ideal gas, which can be modeled by the equation of state. Applying the flux vector splitting approach to the $1 \mathrm{D}$ Euler equations leads to

$$
\mathbf{W}_{t}=\mathbf{F}_{x}=\mathbf{F}_{x}^{-}+\mathbf{F}_{x}^{+}
$$

where the solution vector can be written as $\mathbf{W}=\left(w_{1}, w_{2}, w_{3}\right)^{T}=(\rho, \rho u, E)^{T}$. In order to numerically analyze the 1D Euler system in equation (6), the Jacobian, $\partial \mathbf{F} / \partial \mathbf{W}$, is introduced,

$$
\mathbf{W}_{t}=\frac{\partial \mathbf{F}}{\partial \mathbf{W}} \cdot \frac{\partial \mathbf{W}}{\partial x}=\frac{\partial \mathbf{F}^{-}}{\partial \mathbf{W}} \cdot \frac{\partial \mathbf{W}}{\partial x}+\frac{\partial \mathbf{F}^{+}}{\partial \mathbf{W}} \cdot \frac{\partial \mathbf{W}}{\partial x} .
$$

This leads to an equation including the derivatives of the forward/backward-flux vectors, $\mathbf{F}^{ \pm}$, with respect to the solution vector $\mathbf{W}$. The gradient of the convective flux vectors, $\mathbf{F}^{ \pm}$, is discretized with the discrete forward difference operator, $\underline{\mathbf{D}}_{x}^{+}$, (for the forward-flux) and in backward direction with the discrete backward difference operator, $\underline{\mathbf{D}}_{x}^{-}$, (for the backward-flux),

$$
\begin{aligned}
\mathbf{W}_{t} & =\frac{\partial \mathbf{F}^{+}}{\partial \mathbf{W}} \cdot \frac{\partial \mathbf{W}}{\partial x}+\frac{\partial \mathbf{F}^{-}}{\partial \mathbf{W}} \cdot \frac{\partial \mathbf{W}}{\partial x} \\
& \cong \frac{\partial \mathbf{F}^{+}}{\partial \mathbf{W}} \cdot\left(\underline{\mathbf{D}}_{x}^{+} \cdot \mathbf{W}\right)+\frac{\partial \mathbf{F}^{-}}{\partial \mathbf{W}} \cdot\left(\underline{\mathbf{D}}_{x}^{-} \cdot \mathbf{W}\right)
\end{aligned}
$$


difference operators, $\underline{\mathbf{D}}_{x}^{ \pm}$, are provided in Brehm et al. ${ }^{16}$ For an irregular grid point with an immersed boundary located to the left (right), the optimization procedure is only applied to the differentiation of the forward (backward) flux. From this point forward, it is assumed that for the theoretical discussion the immersed boundary is located to the left. The $n^{t h}$-order accurate finite difference operator for the differentiation of the backward flux is obtained by utilizing $n+2$ grid points (including the boundary point) and applying a least-squares procedure to determine the stencil coefficients for the over-determined system of equations. In order to obtain higher-order accurate pressure and temperature boundary conditions a weighted-least-squares interpolation approach is utilized. More details about the basics of the immersed boundary method can be found in Brehm et al. ${ }^{15,16}$

\section{Viscous Wall Models}

Three different immersed boundary viscous wall extensions are being tested.

\section{III..1. Wall Velocity Deficit Model}

The wall velocity deficit model (WVDM) is based on the idea of reducing the tangential velocity at the wall that would be applied for a slip wall boundary condition in order to mimic the effect of the viscous boundary layer. An important aspect here is that when accounting for the viscous wall boundary conditions on a coarse mesh the resolution is not sufficient to support the large gradients that occur in the viscous boundary layer. In a recent discussion on turbulence modeling by Spalart, ${ }^{28}$ the author pointed out that for the design of computational meshes with wall models used at viscous walls it is crucial to design the mesh correctly so that the gradients in the transition region between the wall model and the near wall mesh can be captured correctly. This aspect is especially problematic for IBMs where a Cartesian grid point can be located infinitesimally close to the viscous wall. In order to obtain the slip wall velocity the tangential velocity is extrapolated with a weighted-least squares extrapolation (WLSQR) stencil,

$$
\boldsymbol{v}_{t}^{*}=\sum_{k=1}^{N} c_{k} \boldsymbol{v}_{t, k},
$$

where $\boldsymbol{v}_{t}^{*}$ is the tangential velocity at the grid line intersection point, $c_{k}$ is the WLSQR interpolation coefficient and $\boldsymbol{v}_{t, k}$ is the tangential velocity at the $\mathrm{k}^{\text {th }}$ grid point within the extrapolation stencil. The velocity vectors at each grid point are locally decomposed into a component normal to the normal vector (tangential to the wall) at the grid line intersection point $\boldsymbol{n}^{*}$ and remainder that is tangential to wall.

$$
\boldsymbol{v}_{t, k}=\boldsymbol{v}_{k}-\left(\boldsymbol{n}^{*} \cdot \boldsymbol{v}_{k}\right) \boldsymbol{n}^{*}
$$

Instead of applying the full slip wall velocity as the boundary condition only a fraction of this velocity is applied.

$$
\boldsymbol{v}_{\partial \Omega}=\beta \boldsymbol{v}_{t}^{*}, \quad \text { with } \quad \beta \in[0,1]
$$

At this point, the model complexity can be controlled through the fractional velocity parameter $\beta$, which can be made a function of the solution vector $\boldsymbol{q}$, the streamwise and wall normal derivatives, the Mach number, the Prandtl number, and the Reynolds number. Different strategies for computing $\beta$ will be assessed. The basic approach has been successfully used to simulate the flow field around the partially dressed nose landing gear from the AIAA BANC-III workshop.

Figure 2 shows the effect of the value of $\beta$ on the pressure distribution around the star board wheel of the landing gear used in the AIAA BANC-III workshop. The pressure distribution for $\beta=0$ (no-slip wall) and $\beta=1$ (slip wall) do not match the experimental data (BART and UFAFF) very well due to predicting separation too early for $\beta=0$ and failing to predict any separation at all for $\beta=1$. A fair match between the numerical simulation and the experimental data can be obtained for an intermediate value of $\beta$.

\section{III..2. Conventional Wall Modeling Approach}

In this approach the wall model by Spalding ${ }^{29}$ is coupled with the IBM discussed in section II.B. The following analytical expression,

$$
y^{+}=u^{+} e^{-\kappa B}\left[e^{\kappa u^{+}}-1-\kappa u^{+}-\frac{\left(\kappa u^{+}\right)^{2}}{2}-\frac{\left(\kappa u^{+}\right)^{3}}{6}\right],
$$




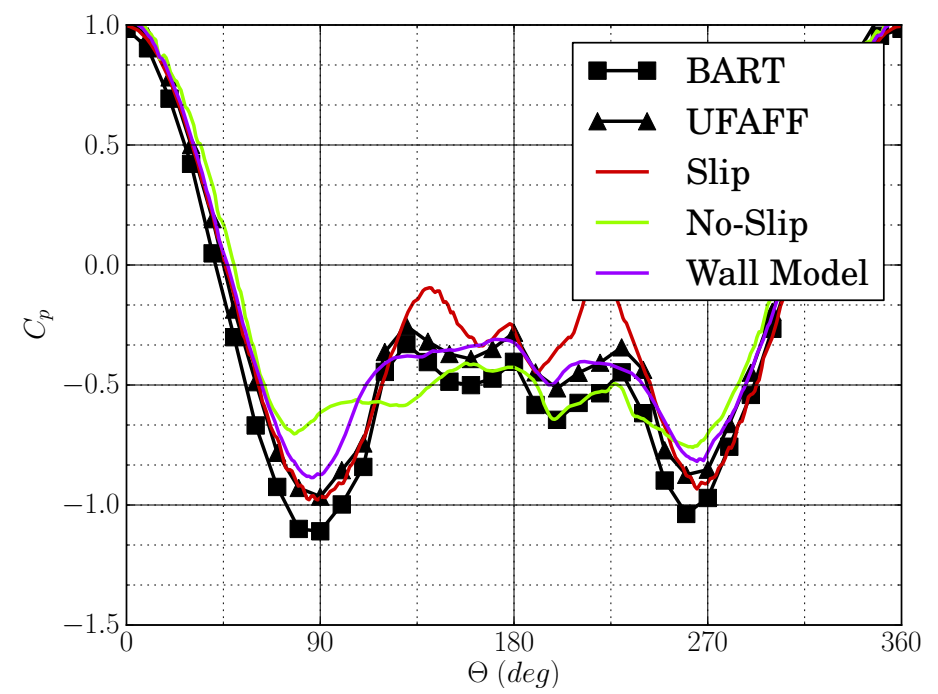

Figure 2. Comparison of pressure distribution around star board wheel of the landing gear used in the AIAA BANC-III workshop considering slip wall, no-slip wall, and wall modeling results. The data is compared to two sets of experimental data (BART and UFAFF).

is valid for the log layer and the sublayer as well as the transition region. The Crocco-Busemann equation is used to obtain the adiabatic wall temperature

$$
T_{w}=T_{r e f}+\frac{r}{2} \frac{v_{t}^{2}}{c_{p}}
$$

where $r$ is the recovery factor, $c_{p}$ represents the specific heat capacity, and $v_{t}$ is the slip wall velocity interpolated from the Cartesian grid. The wall pressure is extrapolated from the outer solution and the wall density is obtained using the ideal gas law. Employing Newton's method the wall shear stress can be iteratively obtained from equation (12). Near the wall, the turbulent eddy viscosity is assumed to follow the relation

$$
\frac{\mu_{t}}{\mu}=\kappa e^{-\kappa B}\left[e^{\kappa u^{+}}-1-\kappa u^{+}-\frac{\left(\kappa u^{+}\right)^{2}}{2}\right] .
$$

As boundary conditions for the turbulence model, we use $k=u_{\tau}^{2} / \sqrt{C_{\mu}}$ for the turbulent kinetic energy and $\epsilon=\rho C_{\mu} k^{2} / \mu_{t}$ for the dissipation rate. The effect of the viscous wall on the outer solution can be modeled by prescribing a reduced slip wall velocity in the same way as for the WVDM or applying a viscous force through viscous flux at the wall. In the final paper, both approaches will be considered and a more detailed discussion of these approaches will be given. The wall modeling approach is similar to the method discussed in Ruffin et al. ${ }^{17}$ whereby different variations are considered on how to transfer information from the wall model to the Cartesian immersed boundary solver.

\section{III..3. Interactive IBL-IBM Approach}

The current integral boundary layer equations are based on previous work by Drela and Giles. ${ }^{30}$ The 2D implementation is used within the rapid aerodynamics analysis tool XFoil. In the final paper, both the 2D and 3D implementations of the interactive IBL-IBM approach will be analyzed. In the current abstract, only the 2D implementation is discussed for the sake of brevity. The IBL method is based on solving the compressible integral momentum and kinetic energy shape parameter equations in the streamwise coordinate direction.

$$
\begin{gathered}
\frac{d \Theta}{d \xi}+\left(2+H-M_{e}^{2}\right) \frac{\Theta}{u_{e}} \frac{d u_{e}}{d \xi}=\frac{C_{f}}{2} \\
\Theta \frac{d H^{*}}{d \xi}+\left(2 H^{* *}+H^{*}(1-H)\right) \frac{\Theta}{u_{e}} \frac{d u_{e}}{d \xi}=2 C_{D}-H^{*} \frac{C_{f}}{2}
\end{gathered}
$$


To model the laminar to turbulent transition process the growth of the most amplified Tollmien-Schlichting wave is tracked (in 2D). The amplitude evolution of the most-amplified TS-wave is modeled as

$$
\frac{d A}{d \xi}=\frac{d A}{d R e_{\Theta}}\left(H_{k}\right) \frac{d R e_{\Theta}}{d \xi}\left(H_{k}, \Theta\right)
$$

In this equation $\frac{d A}{d R e_{\Theta}}\left(H_{k}\right)$ is determined by correlations of spatial growth rates that are pre-computed from solutions to the Orr-Sommerfeld equation, assuming the Falkner-Skan family as baseflow profiles. The OrrSommerfeld equations are solved in the form discussed in Brehm et al. ${ }^{31}$ Once the amplitude $A$ reaches a threshold value of $A_{\text {crit }}$ fully turbulent flow is assumed.

In the turbulent case the shear-stress lag-equation is solved in the form

$$
\frac{\delta}{C_{\tau}} \frac{d C_{\tau}}{d \xi}=5.6\left(C_{\tau E Q}^{1 / 2}-C_{\tau}^{1 / 2}\right)+2 \delta\left(\frac{4}{3 \delta^{*}}\left[\frac{C_{f}}{2}-\left(\frac{H_{k}-1}{6.7 H_{k}}\right)^{2}\right]-\frac{1}{u_{e}} \frac{d u_{e}}{d \xi}\right) .
$$

The three dependent variables for the laminar/transitional flow are the momentum thickness $\Theta$, the displacement thickness $\delta^{*}$, and the TS-wave amplitude $A$. In the turbulent case $C_{\tau}$ is solved for instead of $A$. In order to close the system of equations, involving equations (15-16) and either equation (17) or equation (18), additional functional relations are required. The dependencies used in this work for the laminar and turbulent cases are provided in Drela and Giles. ${ }^{30}$

In the classical integral boundary layer approach the near-wall viscous solution is commonly coupled to the outer solution by the wall transpiration concept which essentially accounts for the boundary layer displacement effect by the transpiration massflow rate $\dot{m} \equiv u_{e} \delta^{*}$. In addition to this approach of coupling the IBM with the IBL equations, a $\beta$ value can be extracted from IBL solution for the WVDM.

\section{Test Cases}

The test cases are chosen to compare the different viscous wall modeling approaches and determine their inherent limitations. The test cases are chosen carefully in order to have reliable data available for the comparison. The degree of complexity is subsequently increased. For all test cases the Cartesian grid solutions are compared to body-fitted unstructured and curvi-linear solutions as well as the available experimental data. Note that the current abstract only presents some preliminary results and a very limited discussion. The final paper will provide a more thorough analysis and a more detailed discussion of the results.

\section{IV.A. Flat Plate Boundary Layer}

The laminar and turbulent flat plate boundary layer flows are the most basic test cases for our viscous wall models. Note that the transition from a symmetry (or slip wall) to a no-slip wall causes a strong adjustment at the leading edge of the plate. The adjustment is very sensitive to the grid resolution in this region and, therefore, the different solutions are compared further downstream of the leading edge. Figure 3 shows a comparison of different immersed boundary methods for the turbulent flat plate boundary layer. For all methods a satisfactory comparison is observed. The symbols represent the solution from LAVA-unstructured considering the SST (red) and SA (green) turbulence models. A more thorough comparison of the different results will be presented in the final paper considering different wall-spacings and discussing the results for the different IBM solutions in more detail.

\section{IV.B. Attached and Separated Flow Past a NACA0012 Airfoil}

The purpose of the next test case is to compare the different methods for a non-zero pressure gradient boundary layer flow. For this purpose, the flow past a NACA0012 airfoil was chosen at different angles of attack. For all solution methods, the pressure distributions and skin friction coefficients are compared. Although, it is well-known that the prediction of the separated flow region is very challenging for RANS, the immersed boundary methods were utilized to simulate the flow around the airfoil up to stall. Figure 4a shows the pressure field around the NACA0012 airfoil at an AoA of $\alpha=0^{\circ}$. The pressure distributions and the skin friction coefficients for body-fitted and the immersed boundary methods are shown in figures $4 \mathrm{~b}$. In the final paper, the results for the different immersed boundary methods will be included. 


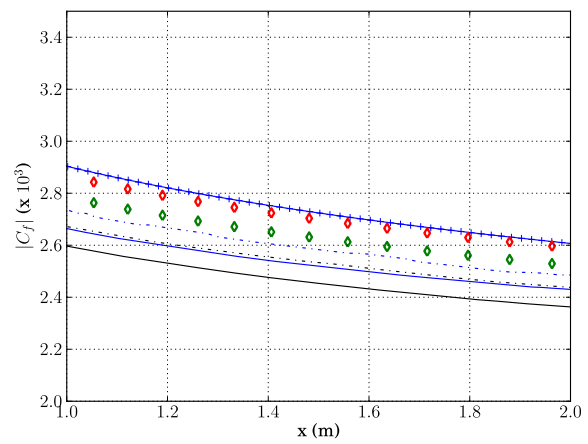

Figure 3. Skin friction coefficient for a turbulent flat plate boundary layer for different body-fitted and IBM solutions.
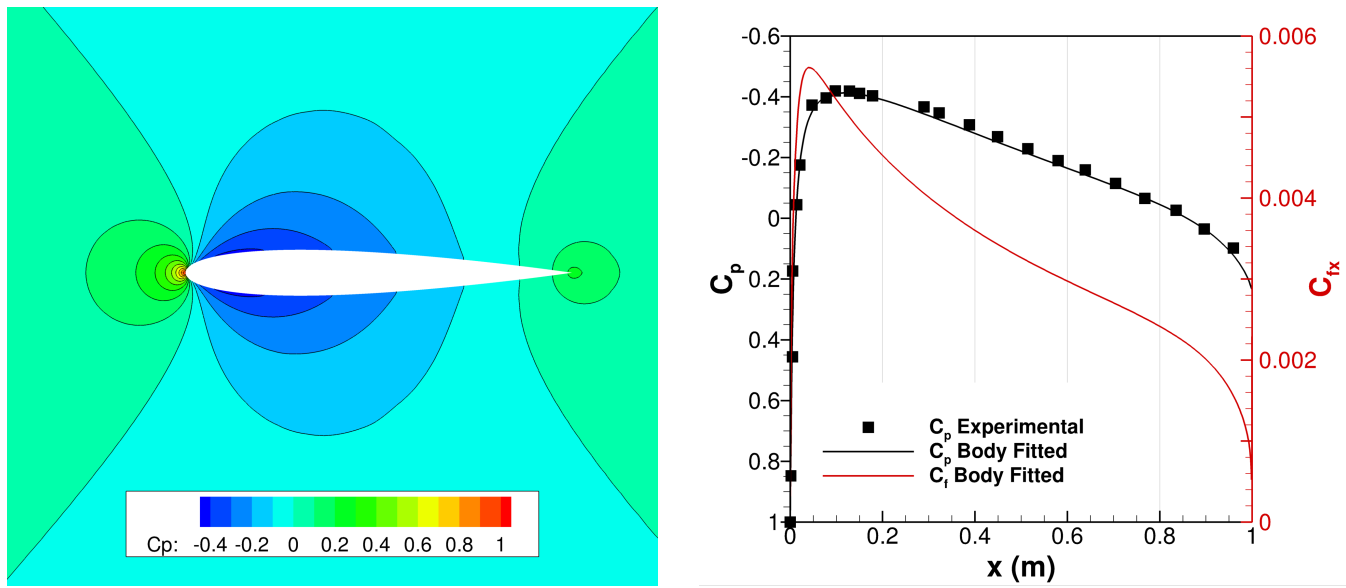

Figure 4. Body-fitted unstructured result for pressure coefficient distribution around NACA 0012 airfoil at Ma $=0.15$ and $R e=6 \times 10^{6}$. IBM results will be added in the final paper.

\section{IV.C. Shock-Induced Separation on RAE2822 Airfoil}

The next test-case introduces an additional challenge when simulating transonic flows. Shock-induced separation occurs for the flow past the RAE2822 airfoil at a critical angle-of-attack. This test-case can be a challenging test case for body-fitted CFD approaches. Figure 5 shows the flow field around the RAE2822 airfoil. In figure 5a, a tail shock can clearly be identified on the suction side of the airfoil. Figure 5b shows the pressure distribution and the final paper will include the skin friction coefficient. The shock on the suction of the airfoil causes a large rise in pressure and a local flow separation $\left(c_{f}<0\right)$ behind the shock. From prior studies it is known that the wall treatment, for example slip wall (as commonly used in Cartesian grid solvers) versus non-slip wall boundary conditions, has a strong effect on the overall pressure distribution. For the final paper, the results for the different immersed boundary methods will be added.

\section{IV.D. Onera M6}

In the last test case the immersed boundary method was applied to the transonic flow past the Onera M6 wing. The pressure distribution on the surface of the wing is shown in figure 6a. Experimental data is only available for pressure distributions at selected spanwise cross-sections marked in figure 6a with grey lines. Figure $6 \mathrm{~b}$ displays a comparison of experimental data with CFD data at the third spanwise cross-section. Our preliminary results employing the different immersed boundary methods indicate that the results are within the range of other CFD solutions obtained with the body-fitted approach (see NASA Langley turbulence modeling website). In the final paper, we will compare the results of all IBM wall modeling approaches with experimental and body-fitted CFD results at all six spanwise locations. Furthermore, the skin friction coefficient will be compared for all computational results. 


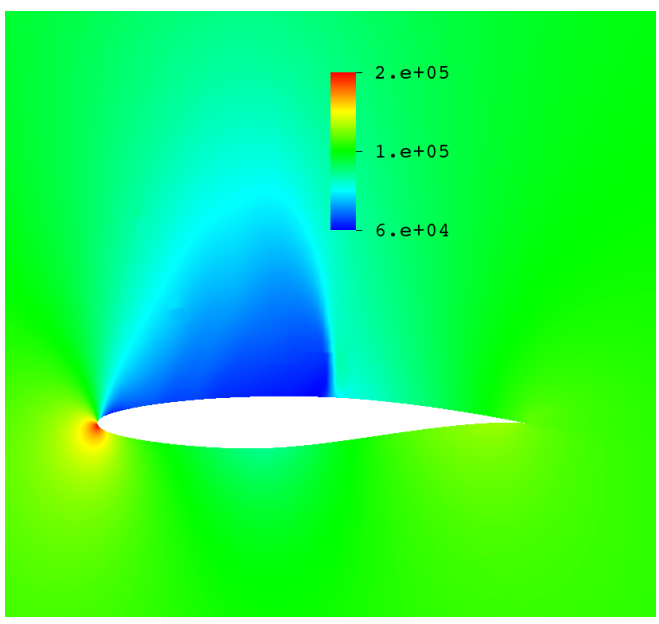

(a)

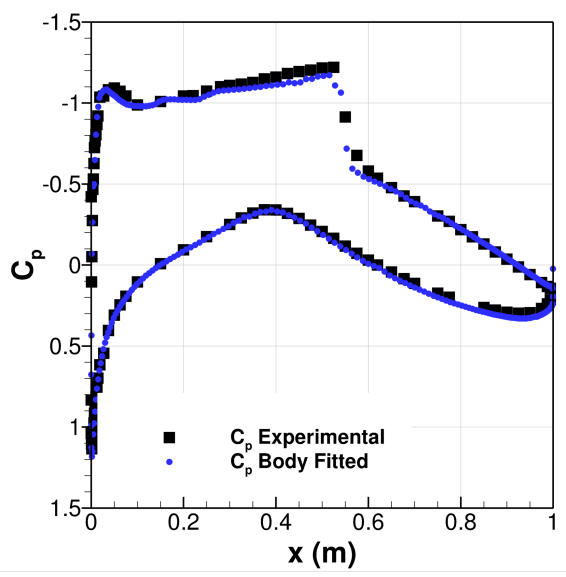

(b)

Figure 5. (a) Pressure field around RAE2822 airfoil and (b) pressure coefficient in comparison to experimental measurement. IBM results will be added in the final paper.

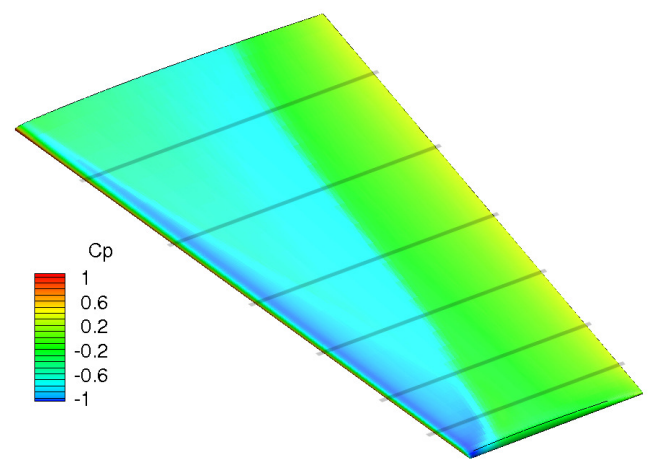

(a)

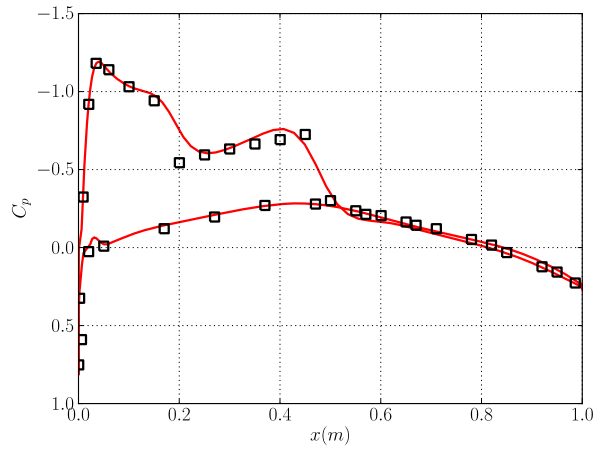

(b)

Figure 6. (a) Pressure distribution on the surface of the Onera M6 wing and (b) pressure coefficient at third spanwise cross-section in comparison to experimental measurement. IBM results will be added in the final paper.

\section{Summary and Outlook}

The current abstract discussed three different viscous wall modeling approaches for immersed boundary methods. While the first two models are solely based on local flow information, the third most promising method utilizes the streamwise history of the boundary layer. The preliminary results (not all shown here) indicate that the IBL-IBM approach has the potential of capturing boundary effects for high-Reynolds number flow simulations. However, it is also certain that the limited scope of test-cases cannot capture all of the challenges immersed boundary methods may encounter for high-Reynolds number flow simulations.

One of the key challenges is to make the IBL-IBM method consistent with the conventional RANS approaches in the limit of $y^{+} \rightarrow 1$. This is a very desirable property to have because it is necessary to obtain well-behaved grid convergence behaviors. The current abstract only provides a limited scope of the final paper. The final paper will provide all the necessary details about the different wall modeling approaches as well as its numerical solution procedures. Furthermore, the different test problems will be described in more detail and the flow conditions will be provided. The final paper will put a strong emphasis on a detailed discussion of the different results and it will analyze the grid convergence properties of these methods. 


\section{References}

${ }^{1}$ Peskin, C., "Numerical Analysis of Blood Flow in the Heart," J. Comput. Phys., Vol. 25, 1977, pp. $220-252$.

${ }^{2}$ Peskin, C. S., "The Immersed Boundary Method," Acta Numerica, Cambridge University Press, 2002, pp. 1-39.

${ }^{3}$ Goldstein, D., Handler, R., and Sirovich, L., "Modeling a Non-Slip Flow Boundary with an External Force Field," J. Comp. Phys., Vol. 105, 1993, pp. 354-366.

${ }^{4}$ LeVeque, R. J. and Li, Z., "The Immersed Interface Method for Elliptic Equations with Discontinuous Coefficients and Singular Sources," SIAM J. Numer. Anal., Vol. 31(4), 1994, pp. 1019-1044.

${ }^{5}$ Wiegmann, A. and Bube, K., "The Explicit-Jump Immersed Interface Method: Finite Difference Methods for PDEs with Piecewise Smooth Solutions," SIAM J. Numer. Anal., Vol. 31(4), 1992, pp. 1019-1044.

${ }^{6}$ Linnick, M. and Fasel, H., "A High-Order Immersed Interface Method for Simulating Unsteady Incompressible Flows on Irregular Domains," J. Comput. Phys, Vol. 204, 2004, pp. 157-192.

${ }^{7}$ Johansen, H. and Collela, P., "A Cartesian Grid Embedded Boundary Method for Poisson's Equation on Irregular Domains," Journal of Computational Physics, Vol. 147, 1998, pp. 60-85.

${ }^{8}$ Mittal, R. and Iaccarino, G., "Immersed Boundary Methods," Annu. Rev. Fluid Mech., Vol. 37, 2005, pp. $239-261$.

${ }^{9}$ Zhong, X., "A New High-Order Immersed Interface Method for Solving Elliptic Equations with Embedded Interface of Discontinuity," Journal of Computational Physics, Vol. 225, 2007, pp. 1066-1099.

${ }^{10}$ Duan, L., Wang, X., and Zhong, X., "A High-Order Cut-Cell Method for Numerical Simulation of Hypersonic BoundaryLayer Instability with Surface Roughness," Journal of Computational Physics, Vol. 229, 2010, pp. 7207-7237.

${ }^{11}$ Kiris, C., Barad, M., Housman, J., Sozer, H., Brehm, C., and Moni-Yekta, S., "The LAVA Computational Fluid Dynamics Solver," AIAA Science and Technology Forum and Exposition 2014, 2014.

${ }^{12}$ Nemec, M. and Aftosmis, M., "Aerodynamic Shape Optimization Using a Cartesian Adjoint Method and CAD Geometry," 24th AIAA Applied Aerodynamics Conference, AIAA 2006-3456, 2006.

${ }^{13}$ Mittal, R., Dong, H., Bozkurttas, M., Najjar, F., Vargas, A., and von Loebbecke, A., "A versatile sharp inerface immersed boundary method for incompressible flows with complex boundaries," Journal of Computational Physics, Vol. 227, 2008, pp. $4825-4852$.

${ }^{14}$ Tezduyar, T., "Finite Element Methods for Flow Problems with Moving Boundaries and Interfaces," Arch. Comput. Methods Eng., Vol. 8, 2001, pp. 83-130.

${ }^{15}$ Brehm, C. and Fasel, H., "A novel concept for the design of immersed interface methods," Journal of Computational Physics, Vol. 242, No. 0, 2013, pp. $234-267$.

${ }^{16}$ Brehm, C., Hader, C., and Fasel, H., "A locally stabilized immersed boundary method for the compressible Navier-Stokes equations," Journal of Computational Physics, Vol. 295, 2015, pp. 475-504.

${ }^{17}$ Ruffin, S. M. and Lee, J., "Adaptation of a k-epsilon Model to a Cartesian Grid Based Methodology," International Journal of Mathematical Models and Methods in Applied Sciences, Vol. 3, 2009, pp. 238-245.

${ }^{18}$ Berger, M. J., Aftosmis, M. J., and Allmaras, S. R., 50th AIAA Aerospace Sciences Meeting, January 2012, AIAA $2012-1301$.

${ }^{19}$ Drela, M. and Giles, M. B., "Viscous-inviscid analysis of transonic and low Reynolds number airfoils," AIAA journal, Vol. 25, No. 10, 1987, pp. 1347-1355.

${ }^{20}$ Aftosmis, M. J., Berger, M. J., and Alonso, J. J., "Applications of a Cartesian mesh boundary-layer approach for complex configurations," 44th AIAA Aerospace Sciences Meeting, January 2006, AIAA 2006-0652.

${ }^{21}$ Rodriguez, D., Sturdza, P., Suzuki, Y., Martins-Rivas, H., Peronto, A., and by Institutions, F., "A Rapid, Robust, and Accurate Coupled Boundary-Layer Method for Cart3D," 50th AIAA Aerospace Sciences Meeting, January 2012, AIAA 2012-0302.

${ }^{22}$ Laible, A. C. and Fasel, H. F., "Temporal Direct Numerical Simulations of Oblique Breakdown for a Cone at Mach 3.5," 49th AIAA Aerospace Sciences Meeting, 2011-0209, 2011.

${ }^{23}$ Zhong, X., "High-Order Finite-Difference Schemes for Numerical Simulation of Hypersonic Boundary-Layer Transition," J. Comp. Phys., Vol. 144, 1998, pp. 662-709.

${ }^{24}$ Visbal, M. and Gaitonde, D., "High-order accurate methods for unsteady vortical flows on curvilinear meshes," AIAA 1998-0131, 1998.

${ }^{25} \mathrm{Shu}, \mathrm{C}$. and Osher, S., "Efficient implementation of essentially non-oscillatory shock-capturing schemes," Journal of Computational Physics, Vol. 77, No. 2, 1988, pp. 439-471.

${ }^{26}$ Brehm, C., Barad, M. F., Housman, J. A., and Kiris, C. C., "A comparison of higher-order finite-difference shock capturing schemes," Computers \& Fluids, Vol. 122, 2015, pp. 184-208.

${ }^{27}$ van Leer, B., "Flux Splitting for the Euler Equations," International Conference on Numerical Methods in Fluid Dynamics, Vol. 170, 1982, pp. 507-512.

${ }^{28}$ Spalart, P. R., "Philosophies and fallacies in turbulence modeling," Progress in Aerospace Sciences, Vol. 74, 2015, pp. 115.

${ }^{29}$ Spalding, D., "A single formula for the law of the wall," Journal of Applied Mechanics, Vol. 28, No. 3, 1961, pp. 455-458.

${ }^{30}$ Drela, M. and Giles, M. B., "Viscous-inviscid analysis of transonic and low Reynolds number airfoils," AIAA journal, Vol. 25, No. 10, 1987, pp. 1347-1355.

${ }^{31}$ Brehm, C., Gross, A., and Fasel, H., "Open-Loop Flow-Control Investigation for Airfoils at Low Reynolds Numbers," AIAA Journal, Vol. 51, No. 8, 2013, pp. 1843-1860. 